\title{
EL IMPACTO DEL GÉNERO Y DE FACTORES SOCIALES EN LA CREDIBILIDAD EMPRENDEDORA DE LOS ESTUDIANTES UNIVERSITARIOS ESPAÑOLES
}

\author{
THE IMPACT OF GENDER AND SOCIAL FACTORS ON THE \\ ENTREPRENEURIAL CREDIBILITY FROM SPANISH UNIVERSITY \\ STUDENTS
}

\author{
Marc Mussons-Torras \\ Doctor por la Universidad Politécnica de Cataluña. \\ Gerente de la Universidad de Vic - Universidad Central de Cataluña, (España). \\ E-mail: marc.mussons@uvic.cat ORCID: http://orcid.org/0000-0001-7972-8149 \\ Elisenda Tarrats-Pons \\ Doctora por la Universidad Politécnica de Cataluña. \\ Profesora de la Facultad de Empresa y Comunicación \\ de la Universidad de Vic - Universidad Central de Cataluña, (España). \\ E-mail: elisenda.tarrats@uvic.cat ORCID: http://orcid.org/0000-0001-7573-2490
}

\section{Citación sugerida:}

Mussons-Torras, M., y Tarrats-Pons, E. (2020). El impacto del género y de factores sociales en la credibilidad emprendedora de los estudiantes universitarios españoles. 3C Empresa. Investigación y pensamiento crítico, 9(2), $133-153$.

https://doi.org/10.17993/3cemp.2020.090242.133-153 


\section{RESUMEN}

La investigación se fundamenta a raíz de los nuevos postulados de la sociedad del conocimiento, denominando a la Unión Europea como una unión por la innovación. Este término engloba directrices y políticas centradas en promover el espíritu emprendedor en las universidades, fomentando a la vez el vínculo con la empresa y el sector público para impulsar un crecimiento sostenible, innovador y de largo plazo. En este sentido, la investigación analiza como el género y determinados factores sociales inciden en el futuro emprendimiento de los estudiantes universitarios. La variable independiente del estudio es la credibilidad del estudiante para emprender, que es el resultado de la combinación de su propia percepción de deseabilidad y de viabilidad, según el modelo del potencial emprendedor de Krueger y Brazeal (1994). La investigación se basa en una muestra de 529 estudiantes españoles de la Universidad de Vic - Universidad Central de Cataluña. En la fase de constructo se establecen los ítems de la variable dependiente según la orientación de la actitud emprendedora de Robinson (1991) y el estudio de Singh y DeNoble (2003). Las conclusiones de la investigación establecen que el género es determinante en la credibilidad emprendedora, dado que persiste en la sociedad un condicionante de carga familiar que afecta al género femenino disminuyendo su credibilidad emprendedora. Pero en cambio, aumenta la credibilidad emprendedora si el estudiante tiene familiares emprendedores, experiencia laboral, y se haya formado en cursos de gestión de empresas.

\section{PALABRAS CLAVE}

Credibilidad emprendedora, Género, Familiares emprendedores, Experiencia laboral, Formación en gestión de empresas. 


\section{ABSTRACT}

The research is based on the new postulates of the knowledge society, calling the European Union as a Union of Innovation. This term involves guidelines and policies focused on promoting the entrepreneurship in the universities, while is increasing the strength link with companies and public sector for the purpose of long-term sustainability growth. In that sense, the research analyzes how gender and social factors influence the entrepreneurship future of university students. The independent variable is entrepreneurship credibility which is built from the mix of desirabiliy and feasibility percepcion according to the entrepreneurial potential model of Krueger and Brazeal (1994). The research is based on a sample of 529 spanish students from the University of Vic - Central University of Catalonia. In the construct phase the items of the dependent variable were being made according to the orientation of the entrepreneurial attitude of Robinson (1991) and to the study of Singh and DeNoble (2003). The conclusions of the research establish the big influence of gender because of family burden, that affects to female reducing their entrepreneurial credibility. And instead, it increases entrepreneurial credibility when the student has family entrepreneurs, working experience or has been trained in business management.

\section{KEYWORDS}

Entrepreneurial credibility, Gender, Family entrepreneurs, Working experience, Business management training. 


\section{INTRODUCCIÓN}

La investigación se fundamenta a raíz de los nuevos postulados de la sociedad del conocimiento, denominando a la Unión Europea como una unión por la innovación. Este término engloba directrices y políticas centradas en promover el espíritu emprendedor. El modelo de la triple hélice de Etzkowitz (Etzkowitz y Leydesdorff, 1998) subraya la importancia de las universidades como uno de los actores principales para el desarrollo de un país. Etzkowitz y Leydesdorff señalaron las interrelaciones necesarias entre las tres esferas: la universidad, la industria y el gobierno o sector público. A mayor interrelación, mayor transformación social y económica a través de la innovación. En especial, se destaca el papel de la universidad en su interacción con la industria. La universidad debe proveer talento, transferir conocimiento y realizar la investigación básica que luego la industria aplicará. Este flujo de conocimiento no es unidireccional, pues también la industria abastece a las universidades con la creación de spin-off, start ups, patentes y modelos de utilidad que muchas veces son propiedad simultánea de universidad y empresa. Todo ello, bajo el denominador común del emprendimiento y de la importancia de que los estudiantes universitarios sean capaces de ir más allá del marco teórico académico. En los postulados de la Europa 2020, del Espacio Europeo de la Educación Superior, del tratado de Bolonia y de los acuerdos de Lisboa se alinean objetivos para potenciar a la universidad como medio en la creación de nuevas empresas, orientando los esfuerzos hacia la innovación en un mundo globalizado, donde Europa debe crecer de forma sostenible y con plena ocupación.

El Espacio Europeo de Educación Superior, según la Declaración de Bolonia (Eurostat, 2009), establece el marco universitario único para que los graduados hagan frente a la demanda de un mercado laboral cada vez más internacional y cambiante que deberá facilitar el crecimiento económico sostenible de la Unión Europea, en adelante UE. En este sentido las universidades han de potenciar la calidad de la investigación, prioridad establecida en la Estrategia de Lisboa (Europeo, 2000), para que la UE pueda ser una de las economías más competitivas y dinámicas del mundo, en lo que se refiere al concepto real de una sociedad basada en el conocimiento. 
La nueva sociedad del conocimiento implicará evolucionar el concepto tradicional de universidad, fomentando el espíritu emprendedor, la transferencia de conocimiento, y la investigación básica. Es necesaria la creación de vínculos cada vez más fuertes entre universidad y industria que servirán para que la universidad lidere nuevas funciones de éxito que supongan un mayor impacto económico, social y cultural (Reichert, 2009). Y sobretodo, es necesario que la universidad rompa sus propias barreras y se convierta en una institución innovadora del conocimiento y no sea solo un centro docente divulgativo de conocimiento como en determinadas épocas del pasado.

\section{DESARROLLO}

El concepto de credibilidad emprendedora se recoge por primera vez en el estudio de Krueger y Brazeal (1994). Según sus autores, la credibilidad emprendedora es la combinación de la percepción de deseabilidad y de viabilidad que hacen que el sujeto, en nuestro caso el estudiante, sea consciente de que la opción de emprender es posible. Su actitud hacia el emprendimiento es creíble, pues lo percibe como deseable y viable.

En el desarrollo de nuestra investigación, seleccionamos como variable dependiente a la credibilidad emprendedora, que es la antesala a la intención de emprender. Es importante destacar que son los autores Krueger y Brazeal (1994) los que elaboran un modelo propio, llamado modelo del potencial emprendedor, y que tiene su origen en una fusión de los dos modelos anteriores de emprendimiento. El modelo del evento emprendedor (Shapero y Sokol, 1982), y la teoría del comportamiento planificado (Ajzen, 1991). En el modelo del potencial emprendedor, el sujeto tiene que tener credibilidad emprendedora. Es decir, debe considerar al emprendimiento como viable y deseable. Sólo entonces podemos decir que el sujeto tiene credibilidad para emprender. En nuestra investigación, consideramos clave poder analizar si existe o no dicha credibilidad, y la cruzaremos con variables sociodemográficas para observar si aumenta o disminuye. Es solo cuando el sujeto tiene credibilidad emprendedora que podemos empezar a creer en un futuro de emprendimiento real. Para ello es necesario que el sujeto llegue al estadio de la intención. 
La intención emprendedora es el punto que antecede a la creación de una empresa, y para ello es necesario que el sujeto tenga propensión a actuar, y se produzca un suceso disparador. En el modelo de Shapero y Sokol se destaca la importancia del suceso disparador como algo inesperado; un despido laboral, un divorcio, o un premio de lotería. La aparición de este evento fortuito e inesperado es lo que acaba generando la necesidad real de emprender un negocio.

Los elementos seleccionados para la variable dependiente de credibilidad emprendedora son del formulario "Entrepreneurial Attitude Orientation" (Robinson, Stimpson, Huefner, y Hunt, 1991), utilizados también en el estudio de Singh y DeNoble (2003) en los ítems n. ${ }^{\circ} 1,3$ y 5”. Los ítems son puntuados por los estudiantes de la muestra según una escala de Likert, donde 0 es totalmente en desacuerdo, 1 en desacuerdo, 2 neutral, 3 de acuerdo y 4 totalmente de acuerdo.

Tabla 1. Composición de los ítems de la variable dependiente.

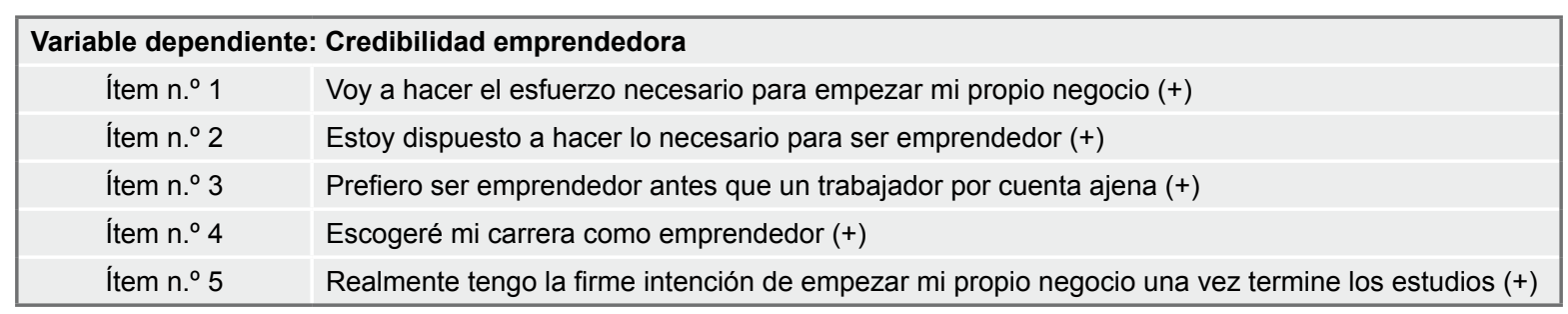

Fuente: elaboración propia.

\subsection{EL GÉNERO}

Numerosas investigaciones ponen de manifiesto la relación entre ser hombre y la intención de crear una empresa (Veciana, Aponte y Urbano, 2005).

En el estudio de cruce de variables demográficas con la creación de empresas, fue contrastado como significativa la relación de que los hombres muestran mayor preferencia por el autoempleo que las mujeres (Kolvereid, 1996). 
Por otro lado, en el estudio efectuado por Veciana y otros (2005), entre los años 1999 y 2000 con una muestra de 18.641 estudiantes universitarios de las universidades catalanas públicas se dio como significativa la relación de dependencia entre el género de los estudiantes y la percepción del deseo de fundar una empresa.

En los estudios de los autores Liñán, Santos y Fernández (2009; 2011), realizados con los datos del GEM data de 2004, sobre una muestra de 33.731 observaciones procedentes de trece países, constata que el género masculino es 1.846 veces más emprendedor que el femenino.

Asimismo en el estudio realizado a estudiantes universitarios americanos de los autores Chen, Green y Crick (1998) se aprecia el mayor interés de los varones hacia la creación de su propio negocio. Sucede lo mismo en el estudio realizado con 904 estudiantes de grados de empresa y ciencias en Grecia (Petridou y Sarri, 2011). También en Austria, en un estudio realizado con 2.124 estudiantes de siete universidades austríacas aparece una mayor intención del genero masculino hacia el emprendimiento (Schwarz, Wdowiak, Almer-Jarz, y Breitenecker, 2009). En Polonia, en un estudio con 253 estudiantes de grados de finanzas y gestión, también se constató una mayor predisposición masculina hacia el emprendimiento (Staniewski y Szopinski, 2015). Y lo mismo sucedió en Alemania, en un estudio con 386 estudiantes de grado (Oehler, Hofer, y Schalkowski, 2015). En Francia, en un estudio con 941 estudiantes procedentes de diversas universidades se dieron los mismos resultados añadiéndose como consecuencia a los aspectos familiares y sociales descritos, un componente alto de aversión al riesgo (Boissin, Branchet, Delanoe, y Velo, 2011). En los distintos informes ejecutivos del Global Entrepreneurship Monitor de España, especialmente a partir de 2006, se hace eco del menor nivel de incorporación de la mujer al emprendimiento. Las razones de esta diferencia entre hombres y mujeres radica en un componente psicosocial relacionado con las percepciones subjetivas que la mujer tiene de la actividad empresarial. Gualquier iniciativa empresarial implica un riesgo más elevado si se la compara con una actividad laboral por cuenta ajena. Este hecho, unido al papel secundario que desde el punto de vista económico 
la mujer ha tenido en una sociedad patriarcal, y su predominancia en el rol familiar, hace que las mujeres lo puedan considerar como una barrera importante para iniciar su propio negocio.

Por lo tanto, el efecto género podría considerarse en un sentido social, donde las mayores cargas familiares de la mujer la limitan en la acción del autoempleo y especialmente cuando requiere un mayor esfuerzo personal de tiempo, factor del que, en general, carece.

Tabla 2. Formulación de hipótesis con la variable del género.

\section{Variable dicotómica independiente: Género}

Hipótesis $n .^{\circ} 1$ El condicionante de carga social y familiar inhibe a la credibilidad emprendedora de las estudiantes de género femenino, a diferencia del género masculino.

Fuente: elaboración propia.

\subsection{ANTECEDENTES FAMILIARES}

Entre los múltiples factores señalados por los investigadores para potenciar el comportamiento emprendedor, los antecedentes familiares se posicionan en un lugar importante. Existen evidencias empíricas de que los emprendedores suelen descender, en gran medida, de familias en las que algunos de sus miembros son auto empleados. De hecho, muchos empresarios han crecido a partir de un patrón desarrollado desde su infancia, adquiriendo conocimientos y comportamientos, además de una visión realista de lo que representa el mundo empresarial. Hay investigaciones y evidencias suficientes en la línea de que el entorno familiar empresarial propicia la aparición de futuros emprendedores.

Crant (1996) demuestra que las personas que tienen un padre empresario son más propensas a expresar intenciones empresariales.

El estudio realizado en la Universidad de Singapur por los autores Wang y Wong (2004), también destaca como variable significativa la existencia de familiares con experiencia en los negocios. 
En Cataluña, en el estudio realizado en las universidades públicas entre los años 1999 y 2000 se demuestra que existe una relación de dependencia entre la variable antecedentes de empresarios en la familia y la firme intención de crear una nueva empresa (Veciana et al., 2005).

Asimismo en el estudio de los autores Wang, Lu y Millington (2011) se constata el efecto positivo de los antecedentes familiares. Concretamente, los progenitores de los estudiantes universitarios de China influyen positivamente en la percepción de deseabilidad hacia el emprendimiento. No obstante, en cambio, no realizan el mismo efecto positivo hacia la percepción de viabilidad, pues no los preparan para la creación de su propio negocio. Hecho que si realizan los progenitores de los estudiantes universitarios de Estados Unidos, donde inciden en la percepción de viabilidad y los preparan adecuadamente para ser capaces de crear su propio negocio (Wang et al., 2011).

Por otro lado, en relación a las variables que integran el denominado "capital social” de la investigación de Honig (2017), constató que el apoyo de amigos y familiares cercanos, así como tener amigos íntimos empresarios o familiares son factores muy significativos para fomentar el emprendimiento. En la misma línea concluye el estudio de los autores Chen, Greene y Crick (1998) destacando que los estudiantes que tienen amigos o familiares emprendedores se muestran más propicios a la creación de su propia empresa.

Tabla 3. Formulación de hipótesis con la variable antecedentes familiares.

\begin{tabular}{|c|c|}
\hline Variable dicotómica independiente: Antecedentes familiares \\
Hipótesis $n .{ }^{\circ} 2$ & $\begin{array}{l}\text { La existencia de familiares empresarios o autoempleados influye positivamente en la credibilidad } \\
\text { emprendedora de los estudiantes universitarios. }\end{array}$ \\
\hline
\end{tabular}

Fuente: elaboración propia.

\subsection{EXPERIENCIA LABORAL PREVIA}

La experiencia laboral permite a la figura del emprendedor adquirir habilidades propias y de gestión que le permitirán en un futuro crear con más efectividad su propia empresa. Esta experiencia nutre al emprendedor de determinadas competencias e información para detectar oportunidades y evaluar riesgos. Es también de gran ayuda para predecir futuros comportamientos emprendedores, incluso en 
los casos en que su anterior experiencia laboral hubiese acabado en fracaso. Con base a lo anterior, se ha demostrado que la experiencia previa de los emprendedores juega un papel importante a la hora de tomar la decisión de crear un negocio. Incluso habiendo realizado un trabajo de base, como aprendiz, que aunque elemental y a veces corto a nivel temporal, le servirá como antecedente válido para su futuro como emprendedor.

Peterman y Kennedy (2003) en su estudio con estudiantes universitarios para conocer el deseo y viabilidad de crear una empresa, verificaron la hipótesis de que aquellos alumnos con más experiencia laboral están también más interesados en fomentar sus conocimientos y experiencias relacionadas con el espíritu emprendedor y buscarán la forma de inscribirse en programas de educación empresarial con el propósito de complementar su idea de negocio. Existe, por lo tanto, según Peterman y Kennedy (2003), una relación positiva entre experiencia laboral previa y deseo y viabilidad de crear su propia empresa.

En el estudio de Honig (2017) relacionado con las variables de capital humano, destacaron como significativas para el emprendimiento la experiencia laboral previa, aunque con un coeficiente de correlación pequeño, sobretodo comparado con los individuos con experiencia previa en start-ups.

También en la investigación realizada en cuatro universidades de Estados Unidos, y otras tres de China, de los autores Wang, Lu y Willington (2011), contrastaron el efecto positivo y significativo de la experiencia laboral previa que incide directamente en la percepción de viabilidad y también, en consecuencia, en la intención emprendedora.

Tabla 4. Formulación de hipótesis con la variable experiencia laboral previa.

\section{Variable dicotómica independiente: Experiencia laboral previa}

Hipótesis $n .^{\circ} 3$ La experiencia previa adquirida en otros trabajos influirá positivamente en la actitud emprendedora de los estudiantes universitarios.

Fuente: elaboración propia. 


\subsection{FORMACIÓN ESPECÍFICA EN EMPRENDIMIENTO}

Los constantes cambios del entorno empresarial sitúan a los conocimientos, la formación técnica, empresarial y de gestión, como factores cada vez más relevantes para los futuros empresarios.

Según la teoría institucional de North (1990) son las instituciones las que condicionan la creación de empresas, porque son las que crean las normas legales que regulan la interacción humana, tanto en relación con las empresas, como en relación a los mercados. Por lo tanto, la creación de nuevas empresas dependerá en gran parte de la capacidad de las instituciones para incentivar acciones que ayuden a crear nuevos emprendedores. De estas instituciones depende también de cómo organizan la formación, de cómo hacen los planes de estudios y de cómo establecen los puentes suficientes entre las Universidades y el tejido empresarial.

También es importante la formación en programas de emprendimiento en los estudios pre-universitarios. En el estudio de Lepoutre, Berghe, Tilleuil y Crijns (2010), en relación a 21 programas educativos de emprendimiento realizados a 3.130 estudiantes de bachillerato en la zona de Flandes, se constató que una vez realizado el programa, hubo un notable incremento en la percepción de deseabilidad, de viabilidad, de la propensión de actuar y, en definitiva, de la intención de crear en el futuro su propio negocio.

Determinados estudios han mostrado la eficacia de la educación en la formación de emprendedores (Drost, 2010). Además, a medida que aumenta el nivel de formación de los potenciales emprendedores, se fortalece el espíritu emprendedor (Robinson et al., 1991).

Así mismo, para la formación de emprendedores es fundamental que se lleve a la práctica un emprendimiento, más que aprender de forma teórica (Eldelman, Manolova, y Brush, 2008).

Dyer (1994) sugiere que los programas específicos de emprendimiento pueden facilitar a los sujetos mejorar su seguridad y confianza, ambas necesarias para empezar con éxito un negocio propio. Muy parecidas son las conclusiones de los autores Krueger y Brazeal (1994) en relación a los efectos directos 
que tienen los programas de emprendimiento en la percepción de viabilidad y autoconfianza de los sujetos que deciden emprender.

Tabla 5. Formulación de hipótesis con la variable de formación en creación de empresas.

\section{Variable dicotómica independiente: Cursos de creación de empresas}

Hipótesis n. ${ }^{\circ} 4$ La participación de los estudiantes universitarios en la materia y cursos sobre creación de empresas influirá positivamente en su intención emprendedora.

Fuente: elaboración propia.

\section{RESULTADOS Y CONCLUSIONES}

La investigación en fase prueba piloto garantizó la validez de contenido y constructo de los ítems de la variable dependiente de credibilidad emprendedora. En la validez de constructo se observaron los índices de fiabilidad para determinar la consistencia de la variable. Para ello se realizó una prueba piloto con 84 sujetos que nos permitió determinar como correlacionaban los ítems con la variable de credibilidad emprendedora. Los resultados definitivos nos permitieron un alfa de cronbach ,881.

La muestra en la fase definitiva reúne los resultados de 529 estudiantes. El 75\% de los participantes son menores de 24 años y el 60\% son mujeres. Además, el 62\% tienen antecedentes familiares empresarios y/o autónomos, el 73\% dispone de experiencia laboral previa, y un 36\% han cursado alguna vez algún tipo de formación vinculada con la creación de empresas.

En el contraste de las hipótesis de las variables dicotómicas, se ha utilizado la prueba U de MannWhitney, que es una prueba no paramétrica aplicada a dos muestras independientes. Es, de hecho, la versión no paramétrica de la habitual prueba t de Student.

En los resultados del contraste de la hipótesis de género se observa como el rango promedio de los valores para los sujetos que son hombres es muy superior al rango promedio de los valores obtenidos por las mujeres. La notable diferencia entre los 287,04 respecto a los 230,66 es lo que dispara el estadístico U de Mann-Whitney y el W de Wilcoxon. Claramente se observa que se rechaza la hipótesis nula de 
que el género no incide en la credibilidad emprendedora a un nivel de significación del 0,000 (p-valor). Por lo tanto, aceptamos la hipótesis n. ${ }^{\circ} 1$ planteada en nuestro estudio, y rechazamos la hipótesis nula de que no existe diferencias entre los dos géneros (Tablas 6 y 7).

Tabla 6. Análisis de rangos.

\begin{tabular}{|c|c|c|c|c|}
\hline \multicolumn{1}{|c|}{} & Genero & N & Rango promedio & Suma de rangos \\
\hline \multirow{2}{*}{$\begin{array}{c}\text { Credibilidad } \\
\text { Emprendedora }\end{array}$} & Masculino & 205 & 287,04 & 58842,50 \\
\hline & Femenino & 301 & 230,66 & 69428,50 \\
\hline
\end{tabular}

Fuente: elaboración propia.

Tabla 7. Estadísticos de contraste Género.

Fuente: elaboración propia.

\begin{tabular}{|c|c|}
\cline { 2 - 2 } \multicolumn{1}{c|}{} & CredEmp \\
\hline U de Mann-Whitney & 23977,500 \\
\hline W de Wilcoxon & 69428,500 \\
\hline Z & $-4,273$ \\
\hline Sig. asintót. (bilateral) &, 000 \\
\hline a. Variable de agrupación: GÉNERO \\
\hline
\end{tabular}

En el contraste de la hipótesis n. 2 (Tablas 8 y 9) observamos que el rango promedio de los valores para los sujetos que tienen familiares empresarios (SI) es muy superior al rango promedio de los valores obtenidos para los que no tienen (NO). La notable diferencia entre los 275,35 respecto a los 226,54 es lo que incrementa el estadístico U de Mann-Whitney y la W de Wilcoxon. Claramente se observa que se rechaza la hipótesis nula de que el hecho de tener familiares empresarios no incide en la credibilidad emprendedora en un nivel de significación del 0,000 (p-valor). Por lo tanto, aceptamos la hipótesis planteada del aumento de la credibilidad emprendedora cuando se tienen antecedentes familiares empresarios. 
Tabla 8. Análisis de rangos.

\begin{tabular}{|c|c|c|c|c|}
\hline \multicolumn{1}{c|}{ Familiares Empresarios } & N & Rango promedio & Suma de rangos \\
\hline \multirow{3}{*}{ CredEmp } & SI & 326 & 275,35 & 89765,50 \\
\hline & NO & 188 & 226,54 & 42589,50 \\
\hline
\end{tabular}

Fuente: elaboración propia.

Tabla 9. Estadísticos de contraste familiares empresarios.

\begin{tabular}{|c|c|}
\cline { 2 - 2 } \multicolumn{1}{c|}{} & CredEmp \\
\hline U de Mann-Whitney & 24823,500 \\
\hline W de Wilcoxon & 42589,500 \\
\hline Z & $-3,601$ \\
\hline Sig. asintót. (bilateral) &, 000 \\
\hline a. Variable de agrupación: FamiliaresEmp \\
\hline
\end{tabular}

Fuente: elaboración propia.

Lo mismo sucede para la hipótesis n. ${ }^{\circ}$ 3, el rango promedio de los valores para los sujetos que tienen experiencia laboral previa es superior al rango promedio de los valores obtenidos por los que no tienen. La diferencia entre los 267,77 respecto a los 229,06 es lo que aumenta el estadístico U de Mann-Whitney y el W de Wilcoxon. Se observa que se rechaza la hipótesis nula de que el hecho de tener experiencia laboral previa no incide en la credibilidad emprendedora a un nivel de significación del 0,01 (p-valor). Por lo tanto, aceptamos la Hipótesis planteada en nuestro estudio, y rechazamos la hipótesis nula de que no existen diferencias entre las dos submuestras (Tablas 10 y 11 ).

Tabla 10. Análisis de rangos.

\begin{tabular}{|c|c|c|c|c|}
\hline \multirow{2}{*}{ ExpLaboralPrevia } & N & Rango promedio & Suma de rangos \\
\hline \multirow{3}{*}{ CredEmp } & SI & 385 & 267,77 & 103092,00 \\
\hline & NO & 130 & 229,06 & 29778,00 \\
\hline
\end{tabular}

Fuente: elaboración propia. 
Tabla 11. Estadísticos de contraste experiencia laboral previa.

\begin{tabular}{|c|c|}
\cline { 2 - 2 } \multicolumn{1}{c|}{} & CredEmp \\
\hline U de Mann-Whitney & 21263,000 \\
\hline W de Wilcoxon & 29778,000 \\
\hline Z & $-2,573$ \\
\hline Sig. asintót. (bilateral) &, 010 \\
\hline a. Variable de agrupación: ExpLaboralPrevia \\
\hline
\end{tabular}

Fuente: elaboración propia.

En relación a la hipótesis n. ${ }^{\circ}$, en la Tabla 12 se recogen los descriptivos de la variable cursos de empresa en relación a la credibilidad emprendedora. La media de los sujetos que tienen cursos de empresa se sitúa entre los 7,94 y los 9,04 (en un 95\% de probabilidad). La mediana en 8 y la media en 8,49. Cabe añadir que hay al menos un sujeto con una puntuación-valor 0 (el mínimo), y al menos un sujeto con puntuación-valor de 15 (el máximo). El rango es pues de 15 (15-0).

En cambio, cuando analizamos a los sujetos que no tienen formación de cursos de creación de empresas, se observa menor puntuación de la variable credibilidad emprendedora ya que la media de los datos se sitúa entre los 6,94 y los 7,67 en un 95\% de probabilidades. Es decir, que si comparamos las dos posiciones dicotómicas observamos que incluso el nivel superior del intervalo de confianza de los que no tienen formación en emprendimiento se encuentra por debajo del nivel inferior de los que sí tienen. Si a ello le añadimos que tanto la mediana $(=7)$, como la media $(=7,31)$ se encuentran también un punto por debajo, parece lógico a priori plantearse que la hipótesis alternativa formulada acabará siendo validada (Tablas 12 y 13). 
Tabla 12. Descriptivos cursos de empresa.

\begin{tabular}{|c|c|c|c|c|c|}
\hline & \multicolumn{3}{|c|}{ CursosEmpresa } & \multirow{2}{*}{$\begin{array}{c}\text { Estadístico } \\
\qquad 8,49\end{array}$} & \multirow{2}{*}{$\begin{array}{l}\text { Error típ. } \\
\qquad, 278\end{array}$} \\
\hline \multirow{26}{*}{ CredEmp } & \multirow{13}{*}{ SI } & \multicolumn{2}{|l|}{ Media } & & \\
\hline & & \multirow{2}{*}{$\begin{array}{l}\text { Intervalo de confianza para la media } \\
\text { al } 95 \%\end{array}$} & Límite inferior & 7,94 & \\
\hline & & & Límite superior & 9,04 & \\
\hline & & \multicolumn{2}{|c|}{ Media recortada al $5 \%$} & 8,51 & \\
\hline & & \multicolumn{2}{|l|}{ Mediana } & 8,00 & \\
\hline & & \multicolumn{2}{|l|}{ Varianza } & 14,624 & \\
\hline & & \multicolumn{2}{|l|}{ Desv. típ. } & 3,824 & \\
\hline & & \multicolumn{2}{|l|}{ Mínimo } & 0 & \\
\hline & & \multicolumn{2}{|l|}{ Máximo } & 15 & \\
\hline & & \multicolumn{2}{|l|}{ Rango } & 15 & \\
\hline & & \multicolumn{2}{|l|}{ Amplitud intercuartil } & 5 & \\
\hline & & \multicolumn{2}{|l|}{ Asimetría } & ,088 & ,177 \\
\hline & & \multicolumn{2}{|l|}{ Curtosis } &,- 890 & ,352 \\
\hline & \multirow{13}{*}{ NO } & \multicolumn{2}{|l|}{ Media } & 7,31 & ,186 \\
\hline & & \multirow{2}{*}{$\begin{array}{l}\text { Intervalo de confianza para la media } \\
\text { al } 95 \%\end{array}$} & Límite inferior & 6,94 & \\
\hline & & & Límite superior & 7,67 & \\
\hline & & \multicolumn{2}{|c|}{ Media recortada al $5 \%$} & 7,30 & \\
\hline & & \multicolumn{2}{|l|}{ Mediana } & 7,00 & \\
\hline & & \multicolumn{2}{|l|}{ Varianza } & 11,164 & \\
\hline & & \multicolumn{2}{|l|}{ Desv. típ. } & 3,341 & \\
\hline & & \multicolumn{2}{|l|}{ Mínimo } & 0 & \\
\hline & & \multicolumn{2}{|l|}{ Máximo } & 15 & \\
\hline & & \multicolumn{2}{|l|}{ Rango } & 15 & \\
\hline & & \multicolumn{2}{|l|}{ Amplitud intercuartil } & 5 & \\
\hline & & \multicolumn{2}{|l|}{ Asimetría } & ,004 & ,136 \\
\hline & & \multicolumn{2}{|l|}{ Curtosis } &,- 542 & ,271 \\
\hline
\end{tabular}

Fuente: elaboración propia.

Nuestro estudio demuestra que haber cursado cursos de creación de empresas se correlaciona con mayor credibilidad emprendedora, validándose la hipótesis (Tabla 13). 
Tabla 13. Estadísticos de contraste cursos de creación de empresas.

\begin{tabular}{|c|c|}
\cline { 2 - 2 } \multicolumn{1}{c|}{} & CredEmp \\
\hline U de Mann-Whitney & 25484,500 \\
\hline W de Wilcoxon & 77810,500 \\
\hline Z & $-3,130$ \\
\hline Sig. asintót. (bilateral) &, 002 \\
\hline a. Variable de agrupación: Cursos Empresa \\
\hline
\end{tabular}

Fuente: elaboración propia.

En conclusión, la investigación está acorde con las investigaciones anteriores mencionadas, constatando que los estudiantes universitarios de género masculino son más emprendedores. Lo mismo sucede en aquellos estudiantes que tienen antecedentes familiares empresarios y también en aquellos con experiencia laboral previa. Realizar cursos de creación de empresas y de emprendimiento como formación específica aumenta la credibilidad emprendedora y puede ser un buen recurso introducirlos en los distintos planes de estudio de las universidades si el objetivo es incentivar el desarrollo económico de un territorio.

\section{REFERENCIAS BIBLIOGRÁFICAS}

Ajzen, I. (1991). The theory of planned behavior. Organizational Behavior and Human Decision Processes, 50(2), 179-211. https://doi.org/10.1016/0749-5978(91)90020-T

Boissin, J. P., Branchet, B., Delanoe, S., y Velo, V. (2011). Gender's perspective of role model influence on entrepreneurial behavioral beliefs. International fournal of Business, 16(2), 182-206. https://www.researchgate.net/publication/267427600_Gender's_Perspective_of_Role_Model_ Influence_on_Entrepreneurial_Behavioral_Beliefs

Ghen, G. G., Greene, P. G., y Grick, A. (1998). Does entrepreneurial self-efficacy distinguish entrepreneurs from managers? Journal of Business Venturing, 13(4), 295-316. https://www. deepdyve.com/lp/elsevier/does-entrepreneurial-self-efficacy-distinguish-entrepreneurs-fromi9VMa8U1As 
Consejo Europeo de Lisboa. (2000). Estrategia de Lisboa. Consejo Europeo. Consejo Europeo de Lisboa.

Drost, E. A. (2010). Entrepreneurial intentions of business students in Finland: implication for education. Advances in Management, 3(7), 28-35. https://ideas.repec.org/a/mgn/journl/v3y2010i7a4.html

Dyer, W. G. J. (1994). Toward a theory of entrepreneurial careers. Entrepreneurship Theory and Practice, 19(19), 7-21. https://doi.org/10.1177/104225879501900202

Eldelman,L.,Manolova,T.,yBrush,G.(2008).EntrepreneurshipEducation:Correspondencebetween Practices of Nascent entrepreneurs and textbook prescriptions for success. Academy of Management Learning and Education, 7(1), 56-70. https://www.jstor.org/stable/40214497?seq=1\#page_scan_ tab_contents

Etzkowitz, H., y Leydesdorff, L. (1998). The triple helix as a model for innovation studies. Science and Public Policy, 25(3), 195-203. https://doi.org/10.1093/spp/25.3.195

Eurostat. (2009). Eurostat statistical book. (Publications_Office, Ed.). European Union.

Honig, B. (2017). Entrepreneurship Education: Toward a Model of Contingency-Based Business Planning. Academy of Management Learning E Education, 3(3), 258-273. https://doi.org/10.5465/ AMLE.2004.14242112

Jose, F., Liñan, F., y Santos, J. (2009). Cognitive Aspects of Potential Entrepreneurs in Southern and Northern Europe: an Analysis Using GEM-Data. Revista de Economía Mundial, 23, 151-178. https://idus.us.es/xmlui/handle/11441/16460

Kolvereid, L. (1996). Prediction of employment status choice intentions. Entrepreneurship Theory and Practice, 21(1), 47-57. https://www.scirp.org/(S(lz5mqp453edsnp55rrgjct55))/reference/ ReferencesPapers.aspx?ReferenceID=2101137 
Krueger Jr, N. F., y Brazeal, D. V. (1994). Entrepreneurial Potential and Potential Entrepreneurs. Entrepreneurship Theory and Practice, 18(3), 91-104. https://papers.ssrn.com/sol3/papers. cfm?abstract_id $=1505244$

Lepoutre, J., Van den Berghe, W., Tilleuil, O., y Crijns, H. (2010). A new approach to testing the effects of entrepreneurship education among secondary school pupils. Vlerick Leuven Gent Management School Working Paper Series 2010/01. https://www.researchgate.net/publication/46469868_A_ new_approach_to_testing_the_effects_of_entrepreneurship_education_among_secondary_ school_pupils

Liñán, F., Santos, F.J., y Fernández,J. (2011). The influence of perceptions on potential entrepreneurs. International Entrepreneurship and Management Fournal, 7(3), 373-390. https://doi.org/10.1007/ s11365-011-0199-7

North, D. (1990). Institutions, Institutional Change and Economic Performance. Cambridge University Press.

Oehler, A., Hofer, A., y Schalkowski, H. (2015). Entrepreneurial education and knowledge: empirical evidence on a sample of German undergraduate students. Fournal of Technology Transfer, 40(3), 536-557. https://doi.org/10.1007/s10961-014-9350-2

Peterman, N. E., y Kennedy, J. (2003). Enterprise Education: Influencing Students' Perceptions of Entrepreneurship. Entrepreneurship Theory and Practice, 28(2), 129-144. https://doi.org/10.1046/ j.1540-6520.2003.00035.x

Petridou, E., y Sarri, K. (2011). Developing “potential entrepreneurs” in higher education institutes. Fournal of Enterprising Culture, 19(1), 79-99. https://doi.org/10.1142/S0218495811000647

Reichert, S. (2009). Institutional diversity in European higher education. European University Association. 
Robinson, P. B., Stimpson, D. V, Huefner, J. C., y Hunt, H. K. (1991). An attitude approach to the prediction of entrepreneurship. Entrepreneurship Theory and Practice, 15(4), 13-31. https://doi. org/10.1177/104225879101500405

Schwarz, E., Wdowiak, M., Almer-Jarz, D., y Breitenecker, R. (2009). The effects of attitudes and perceived environment conditions on students entrepreneurial intent. Education and Training, 51(4), 272-291. https://doi.org/10.1108/00400910910964566

Shapero, A., y Sokol, L. (1982). The social dimension of entrepreneurship. En C.A. Kent, D.L. Sexton, y K.H. Vesper (Eds.), The encyclopedia of entrepreneurship. Prentice H.

Singh, G., y De Noble, A. (2003). Views on self-employment and personality: an exploratory study. Fournal of Developmental Entrepreneurship, 8(3), 265-281. https://www.researchgate.net/ publication/288218612_Views_on_self-employment_and_personality_An_exploratory_study

Staniewski, M. W., y Szopinski, T. (2015). Student readiness to start their own business. Economic Research-Ekonomska Istrazivanja,28(1),608-619. https://doi.org/10.1080/1331677X.2015.1085809

Veciana, J. M., Aponte, M., y Urbano, D. (2005). "University students" attitudes towards entrepreneurship: A two countries comparison. The International Entrepreneurship and Management, 1(2), 165-182. https://doi.org/10.1007/s11365-005-1127-5

Wang, C. K., y Wong, P. K. (2004). Entrepreneurial interest of university students in Singapore. Technovation, 24(2). https://doi.org/10.1016/S0166-4972(02)00016-0

Wang, W., Lu, W., y Millington, J. K. (2011). Determinants of Entrepreneurial Intention among College Students in China and USA. Fournal of Global Entrepreneurship Research, 1(1), 35-44. https:// pdfs.semanticscholar.org/9800/5ce764bed15d419ef07edc6f589068237ad9.pdf 
